\title{
Impact Response Characterization as basis for Bearing Diagnostics and Prognostics
}

\author{
Andrea Sanchez Ramirez ${ }^{1}$, Richard Loendersloot ${ }^{1}$, \\ Tiedo Tinga ${ }^{1,}$ Giuseppe D’Angelo ${ }^{2}$ \\ ${ }^{1}$ Dynamics Based Maintenance, University of Twente, The Netherlands \\ Building Horst, Room N135. Phone 31534893405/ +31534892460 \\ P.O. Box 217, 7500 AE Enschede. The Netherlands \\ 2 Process Research, Centro Ricerche Fiat, Italy \\ Strada Torino 50, 10043 Orbassano, Italy \\ a.sanchezramirez@utwente.nl
}

\begin{abstract}
Impact response due to sudden changes on the contact forces is an intrinsic phenomenon at all stages of the bearing life. Even prior to the actual occurrence of surface damage, short duration impacts occur in the case of insufficient lubrication where asperities or particles interact with the surface of its mating element. As the surface condition deteriorates, the energy released by the impacts increases affecting structural modes. As soon as the system condition worsens, lower frequencies are excited and consequently increased vibration levels are displayed. The impulse response function reflects the modulation phenomena between contact forces, structural resonances and driving forces taking place in the bearing and the mechanical system that contains it. The characterization of the components of the impulse response becomes then a valuable tool for vibration monitoring.

This article is framed within the research on development of advanced vibration monitoring systems, for which the authors follow a systemic approach in the definition of the monitoring requirements based on the function-failure analysis of the monitored object. The discussion focuses on the correspondence between the monitoring and monitored systems, by analyzing the impulse behavior as illustrated by the train bearings. The comparative analysis between environment and damage induced impacts highlights the different stages in bearing damage.
\end{abstract}

Keywords: Impact response, bearing diagnostics and prognostics, high frequency sampling.

\section{Introduction}

On the development of Vibration Monitoring Systems (VMS), the authors (Sanchez et al, 2013) have proposed a physics-based decision support framework for the definition of monitoring strategies and the design of advanced monitoring systems. The framework follows a Function-Behavior-Structure (FBS) ontology, which defines three high-level categories for the properties the monitoring systems. The assigned

adfa, p. 1, 2011.

(C) Springer-Verlag Berlin Heidelberg 2011 
Functions of the monitoring system focus on damage characterization by: i) detecting the existence of damage, ii) trying to explain -diagnose- the origins of such damage and iii) providing a sensible prognostics of the remaining useful life based on the damage evolution. The Behavior stage makes use of damage features immersed on the vibration signal to support the fulfillment of the selected functions. Finally, the Structure level relates to the physical implementation of the monitoring system in the context of innovative technologies for sensing, processing and communication.

Implicit in the framework physics-based character lays the need for synthetizing the monitored object complexity into differentiable damage attributes, which define the behavior stage of the monitoring system. To this end, the monitored object is also subject of a functional-failure analysis. The agreement among the dynamics description of the monitored system and the behavior layer of the monitoring system forms a key aspect to ensure the success of VMS.

This article presents the impulse response as a damage feature for bearing damage characterization. The choice is to focus on impact loading is based on the acknowledgment that impulsive loads are present throughout the entire bearing life as presented in will be shown in the first section of the paper. The second section discusses the implications for the monitoring system in relation to the functions of detection, diagnostics and prognostics. The case study concerns to the comparative study between environment-induced and damage-induced impacts for railway bearing. Final conclusions are presented.

\section{Bearing : Functional - Failure}

The physics-based character of the framework supports the processes of analyzing the monitored object and reducing its complexity by damage features. The functionalfailure approach takes the system functionality as reference to define its failure process; therefore failure is defined as the loss of functionality ${ }^{1}$, described by its failure modes and explained by the failure mechanisms. Following this approach, bearings are defined by two functions: i) enabling free rotation between two components with different rotation speed, and ii) ensuring structural support for the correct distribution of the concurrent forces on the rotating component. Fig. 1 illustrates function-failure analysis for the bearing at the different levels the failure takes place, f.i. local, component and application level.

\subsection{Bearing Functions}

The free rotation function takes place at the contact zone and it is influenced by the surface quality, material stiffness and lubricant properties.

\footnotetext{
${ }^{1}$ Failure has a different connotation than Damage. The first term is used in the context of the monitored system, while the later emphasizes on the monitoring and maintenance implications. Furthermore, Failure implies a binary condition, whether the system fulfills its function or not. Whereas damage is presented as a continuous of the deterioration process of the component.
} 


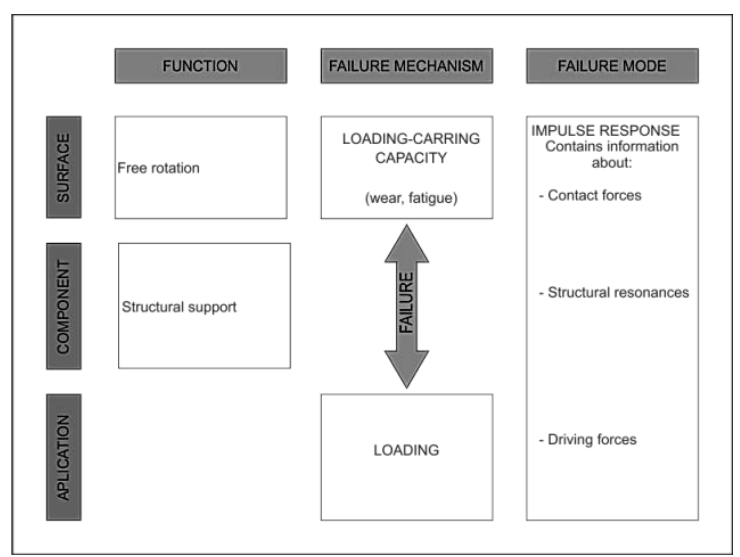

Fig. 1. Function-Failure Analysis for bearings

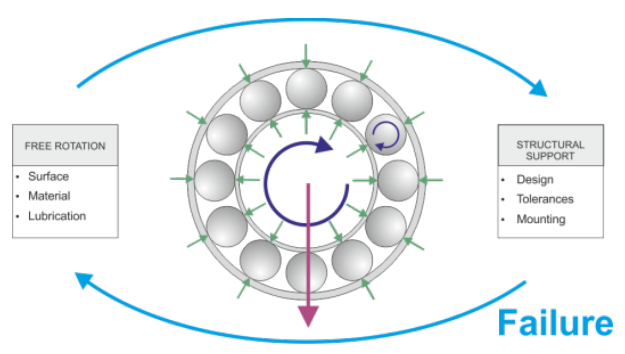

Fig. 2. Function interaction due to failure

Moreover bearing life is strongly related to the magnitude of the forces taking place at the contact zone. High contact forces can be caused by vibration on the bodies, variations of the lubrication film thickness and surfaces asperities (Wijnant, 1998).

Although the interaction among these phenomena is not fully defined, there is a general consensus that such reasons determine the type of failure mechanism(s) most likely to develop in the material, such as fatigue, wear or fretting corrosion. At the component level, the structural support function displays a strong morphological nature since the bearing configuration -design, mounting and tolerances- has to adapt to the prescribed load distribution requested for the application. From the design perspective, the free rotation and structural support functions may seemingly rely on two independent parameter sets. However their interaction appears stronger throughout the bearing deterioration, both at the local and component level (Fig. 2). 


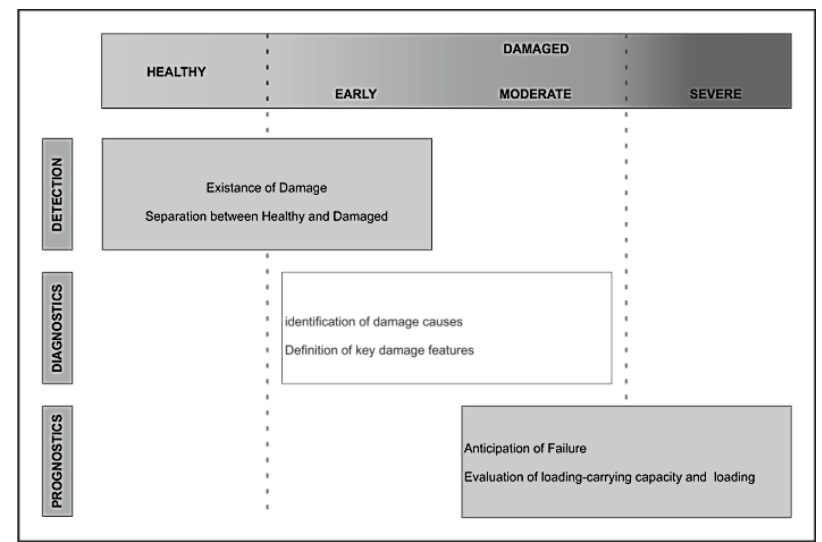

Fig. 3. Healthy-Damage state of bearings versus VMS functions

\subsection{Bearing Failure Response}

The starting point of the discussion on bearing dynamics (behavior) is by recognizing that that even under perfect conditions, bearings are an intrinsic source of vibrations because of the periodical variation of the contact stiffness (Liew and Lim, 2005).

Local superficial defects, such as superficial cracks or added material due to overrolled wear, cause abrupt changes on the contact load which generates short duration pulses of very high frequency content. As possible consequences for the bearings, these pulses can cause wear at the surface, or fatigue increasing crack growth.

The symptoms of the developing failure mechanism, or failure modes, also display distinctive features on the bearing impulse response. As the surface condition deteriorates, the energy released by the impacts increases and starts impacting the bearing structure, therefore lower frequencies are excited and consequently increased vibration levels are displayed. When the occurrence of the impacts is low, the bearing restores its original state by means of the damping. However, if the bearing has not restored completely while another significant impact occurs, the leading edge of the new impact response is buried in the delay of the previous impact. This superposition of impact responses turns into higher overall vibration levels with higher stochastic behavior. In consequence, the impulse response characterization provides significant information for the evaluation of bearing damage.

\section{$3 \quad$ Monitoring System : Functional Analysis}

While the VMS functions are of incremental nature, this does not imply that all VMS have to fulfill the detection, diagnostics and prognostics roles. Detection function can be understood as the (binary) differentiation between the healthy and damaged status for the component. Since impact loading is intrinsic of the entire bearing life, there are not generally accepted criteria for defining whether a bearing is in a damaged condition. Diagnostics deals with the identification of the damage underlying causes 
via the processing of the vibration signal. To this end, the vibration signal is decomposed into meaningful damage features for linking the expected failure modes and failure mechanisms. Prognostics focuses on the anticipation of failure by the definition of tolerable damage before the system is unable to fulfill its functions. This implies the evaluation of the actual loading and loading-carrying capacity associated to the ongoing failure mechanisms towards the quantification of remaining useful life ( Fig. 3).

\subsection{Impact response Analysis using Empirical Mode Decomposition (EMD)}

The bearing vibration displays a high non-stationary behavior because of the contact-stiffness variations and continuous impact loading. Therefore it is ought to be described more accurately by their instantaneous frequency (IF) as a time-varying parameter. The Hilbert-Huang Transform (HHT) is proposed for impact characterization because of its adaptive nature that avoids the restrictions imposed by stationary methods. The HHT comprises two phases, firstly the signal is processed through the Empirical Mode Decomposition (EMD) that returns a collection of Intrinsic Mode Functions (IMFs), subsequently for each IMF the spectral content is investigated via the Hilbert transform. Further enhancement of the HHT is proposed by the IMFs orthogonalisation, in order to ensure the accurate re-composition of the original time signal. More accurate energy content distribution is achieved by the Lagrangian-like Teager-Keiser Energy Operator (TKEO), which enhances low frequency modulations of the vibration signal (Cisi. 2013).

\section{Case Study: Train Bearings}

Train bearings are used to illustrate the differences between damage-related and environment impacts. From the VMS perspective, train bearings present several challenges because of the difficulty in separating the influence of operation (weight, speed), environment (wheel-rain interaction) and the bearing condition itself. Bearings for railways are designed with special considerations in lubrication and tolerances for compensate the high impulsive forces due to the wheel-rail disruptions (Railways SKF, 2012). Two similar bearings (Bearing A and Bearing B) are compared from a train moving at $5.43 \mathrm{~m} / \mathrm{s}$ (Fig. 4). The characteristics of the bearings are provided in the Table 1.

Table 1. Bearing characteristics and Failure Frequencies

\begin{tabular}{l|l|l|l}
\hline \multicolumn{2}{c|}{ Inputs } & \multicolumn{2}{c}{ Failure Frequencies } \\
\hline Bearing CRB type SKF & & Shaft Speed Frequency & $2,192 \mathrm{~Hz}$ \\
Sampling Frequency & $25 \mathrm{KHz}$ & Inner Race Defect (BPFI) & $26.081 \mathrm{~Hz}$ \\
Pitch Diameter & $136 \mathrm{~mm}$ & Outer Race Defect (BPFO) & $19.944 \mathrm{~Hz}$ \\
Train Speed & $5.43 \mathrm{~m} / \mathrm{s}$ & Ball Spin Frequency (BSP) & $8.073 \mathrm{~Hz}$ \\
\hline
\end{tabular}



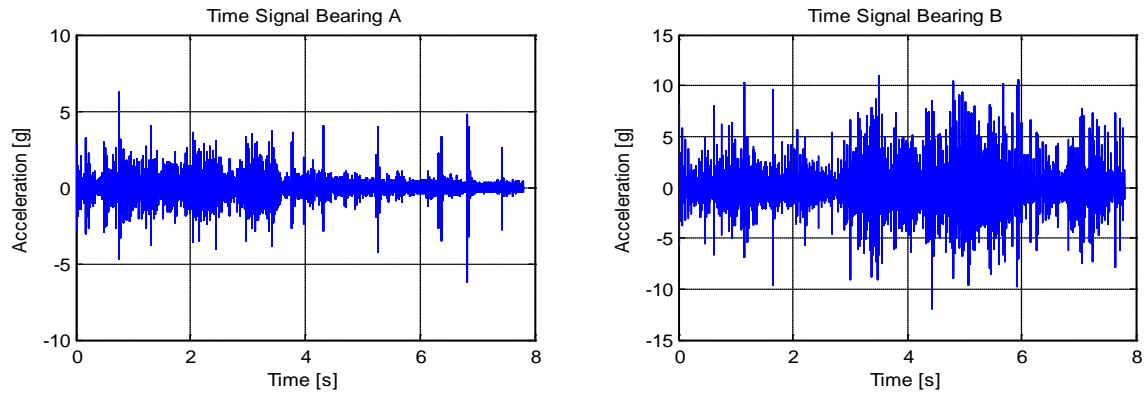

Fig. 4. Bearing $A$ and Bearing $B$
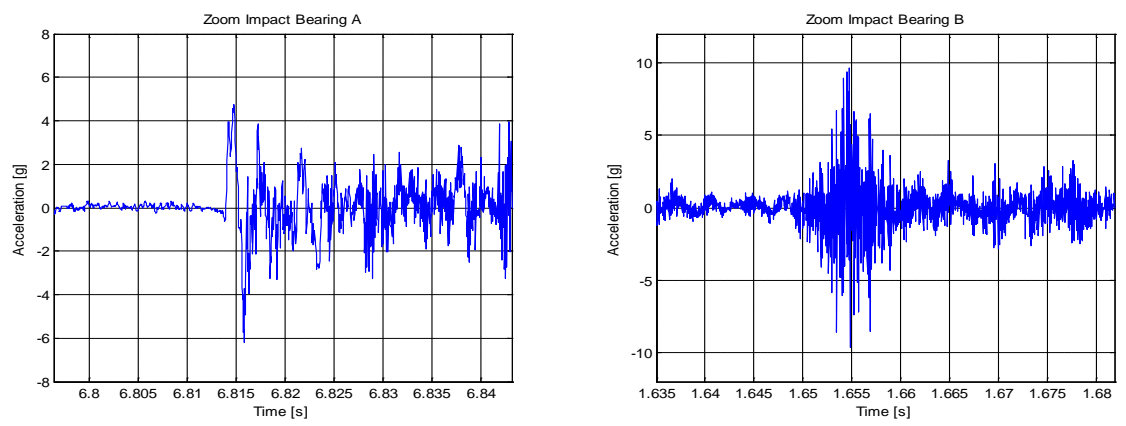

Fig. 5. Zoomed signal for impact analysis.
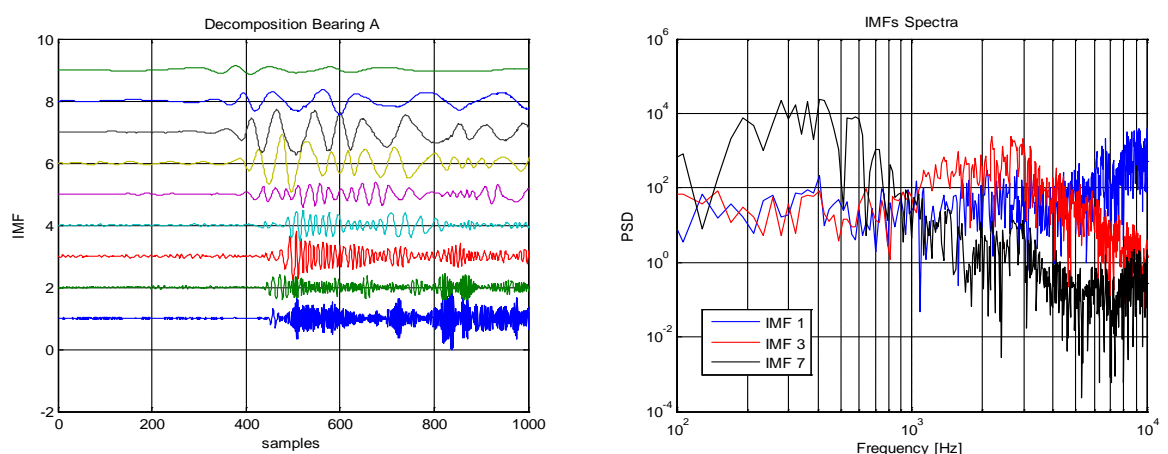

Fig. 6. Bearing A. IMF decomposition and spectra.

Figure 5 shows the extracted impulse to base the impulse analysis. Fig. 6 shows the IMFs for bearing A for the time decomposition (left) and the spectra of selected IMFs(right). The time representation allows an acute visualization of the crossing over a surface disruption. The delay between the lower and higher IMFs is explained by excitation of the structural modes prior the disturbances on the contact forces as reported by Sawalhi and Randall (2011). 

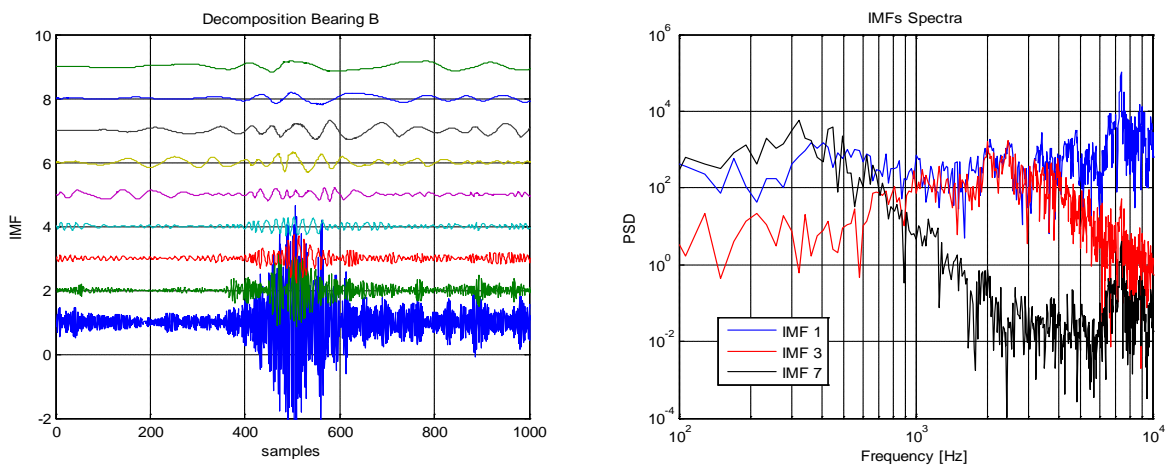

Fig. 7. Bearing B. IMF decomposition and spectra.
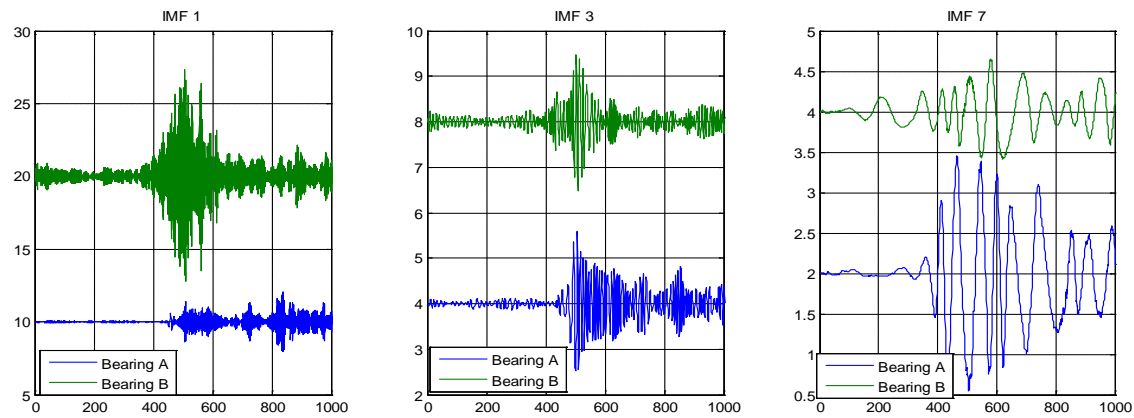

Fig. 8. Comparison IMFs 1,3,7 for bearing A and bearing B.
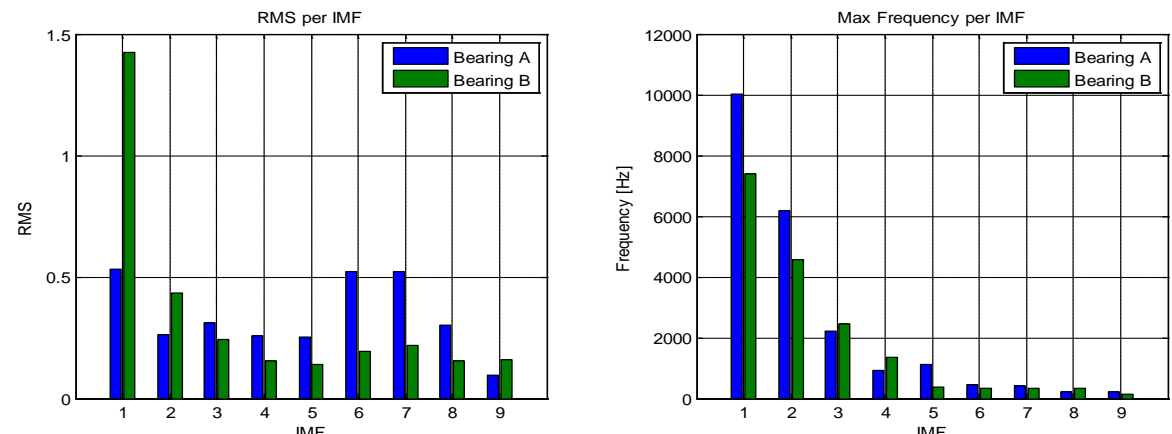

Fig. 9. RMS and Max Frequency per IMFs.

Fig. 6 (right) shows how the IMFs adapt to different frequency bands. Similar decomposition for Bearing B is presented in Fig. 7, for which is seen that the first IMF is the most excited. 

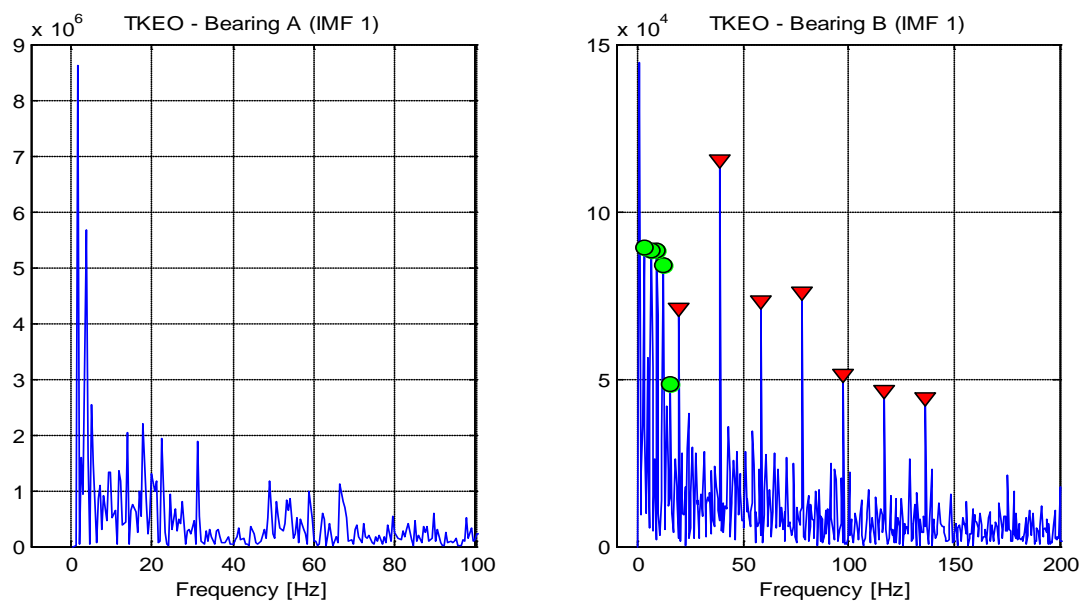

Fig. 10. TKEO for IMF 1 for Bearing A and Bearing B.

A comparison between Bearing A and B for IMFs 1, 3 and 7 is presented in Fig. 8, which highlights the contrast between the low and high frequency content respectively. Fig. 9 presents the IMF summary for rms and maximum frequency. Bearing A presents an even distribution of energy across the different IMFs, contrary to Bearing $\mathrm{B}$ for which the most energy is extracted at the IMF 1 . The frequency distribution per IMFs follows similar trend for both signals. This overview confirms that Bearing B is likely to display a localized damage since the impact energy is mainly related to high frequencies. In comparison, for Bearing A the energy is distributed more evenly, displaying similar values for the high and low frequency band.

After the analysis of the individual impact, the complete signals (Fig. 4 ) are analyzed combining EMD and TKEO, as presented in Fig. 10. The use of longer observation period in combination with TKEO aims at finding the repetivite patterns linked to the events observed in the individual impacts by enhancing the energy content within the vibration signal. For Bearing A there is not specific features observed in the spectrum. Instead, for Bearing B two harmonic families are observed. The one with the lowest frequecy of $3.072 \mathrm{~Hz}$ and its harmonics (green circular markers), and the second of $19.97 \mathrm{~Hz}$ and its harmonics (red triangular markers). The calculated wavelegth for the $3.072 \mathrm{~Hz}$ at the given train speed equals $0.48 \mathrm{~m}$, which is likely to be related to wheel circumpherence abnormalities or rail corrugations. The second harmonic band is related to the Outer Race Defect (BPFO) as presented in Table 1. 


\section{Conclusions}

The approach proposed makes used of a functional analysis for analyzing the bearing failure in terms of failure mode and failure mechanism. This approach is linked to the functions of detection, diagnostics and prognostics of the monitoring system. The impulse response function is chosen as damage feature based on its likelihood to reveal information about the contact and driven forces, and structural resonances during the impact. The use of EMD was proven effective to separate the main modes of the impulse signal.

The analysis of the train bearings illustrate the different nature between environment-induced and damaged-induced impact response. While damage induces disturbances at the contact forces with highest frequency content, environment affects mainly the lower frequencies related to the main driving forces of the application, in this case the wheel-race contact. However, if contact forces, structural resonances and driving forces are affected simultaneously, it is likely that the bearing experience a failure. In a general sense, severe failure at the free rotation function may lead to instability of the component dynamics, and therefore the structural support function. On the opposite sense, deviations in the loading distributions different to the nominal specifications are likely to accelerate the failure mechanism at the local level.

For early detection of bearing damage the first IMF was shown sufficient since it relates to the disturbances in contact forces linked to the free rotation of the bearing. The evaluation of both free rotation and structural support, higher number of IMFs to are required to extract information on the structural resonances. The diagnostics of the underlying causes of the detected damage (Race Damage) and external factors (repetitive forcing frequencies) was achieved by using long observation periods and enhanced energy operator.

\section{Acknowlegdments}

The research leading to these results has received funding from the European Union Seventh Framework Programme FP7/2007-2013 under grant agreement $n^{\circ} 289041$. WIBRATE "Wireless, Self-Powered Vibration Monitoring and Control for Complex Industrial Systems". Special thanks to Centro Ricerche Fiat S.C.p.A and Perpetuum Ltd for providing the vibration data used for validation.

\section{$7 \quad$ References}

Sanchez Ramirez, A; Loendersloot, R., Jauregui, J. M., \& Tinga, T. (2013). Design Framework for Vibration Monitoring Systems for Helicopter Rotor Blade Monitoring using Wireless Sensor Networks. In International Workshop on Structural Health Monitoring (pp. 1023 - 1030). September 10-12. Stanford University. California.

Tinga, T. (2013). Principles of Loads and Failure Mechanisms. London: Springer London. doi:10.1007/978-1-4471-4917-0 
Lugt, P.M. (2012). Grease Lubrication in Rolling Bearings. New York: John Wiley \& Sons. ISBN: 978-1-118-35391-2

Wijnant. Y.H. (1998). Contact Dynamics in the field of Elastohydrodynamic Lubrication. Ph.D. Thesis. University of Twente. The Netherlands.

Liew and Lim (2005). Analysis of time-varying rolling element bearing characteristics. In Journal of Sound and Vibration 283. Pg 1163-1179.

Cisi, A., Zanella, A., D’Angelo, G,. (2013). Rolling Element Bearing Fault Detection Based on Orthogonal Hilbert-Huang Transform, In Tenth International Conference on Condition Monitoring and Machinery Failure Prevention Technologies, CM and MFPT, June 18-20, Poland

Railways SKF (2012). Bearing designs Cylindrical roller bearings and units. Railway technical handbook, Vol 1, Ch 4, Pg 88- 95.

Sawalhi, A., Randall. R. B. (2011). Vibration response of spalled rolling element bearings: Observations, simulations and signal processing techniques to track the spall size. In Journal Mechanical Systems and Signal Processing 25. Pg 846-870. 